\title{
Relation of Elevated Serum Uric Acid Level to Endothelial Dysfunction in Patients with Acute Coronary Syndrome
}

\author{
Yuichi Saito, Hideki Kitahara, Takashi Nakayama, Yoshihide Fujimoto and Yoshio Kobayashi
}

Department of Cardiovascular Medicine, Chiba University Graduate School of Medicine, Chiba, Japan

Aim: Serum uric acid (SUA) level is known to have a prognostic value in patients with acute coronary syndrome (ACS). Endothelial function plays an important role in the development of cardiovascular disease. Although relation between SUA level and endothelial function has been previously studied in various populations, it is partially understood in patients with ACS.

Methods: A total of 55 patients with ACS with measurements of SUA level and reactive hyperemia index (RHI) to evaluate endothelial function were included. They were classified into three groups according to the tertiles of SUA level. The tertiles of SUA level were as follows: low tertile, $\leq 5.2 \mathrm{mg} / \mathrm{dl}$; intermediate tertile, 5.3 to $6.5 \mathrm{mg} /$ $\mathrm{dl}$; and high tertile, $\geq 6.6 \mathrm{mg} / \mathrm{dl}$.

Results: Mean SUA level and RHI were $5.8 \pm 1.5 \mathrm{mg} / \mathrm{dl}$ and $1.88 \pm 0.58$. There was a significant negative correlation between SUA level and RHI $(r=-0.41, p=0.002)$. RHI was stepwisely observed in favor of the higher tertile groups $(2.14 \pm 0.74$ vs. $1.84 \pm 0.45$ vs. $1.67 \pm 0.38, p=0.04)$. Multivariate analysis showed elevated SUA level as an independent predictor of reduced RHI.

Conclusion: Elevated SUA level was significantly associated with endothelial dysfunction in patients with ACS, possibly leading to subsequent poor outcomes following ACS.

Key words: Uric acid, Endothelial function, Reactive hyperemia index, Acute coronary syndrome

\section{Introduction}

Several epidemiological studies have shown a relation between elevated serum uric acid (SUA) level and subsequent cardiovascular events, including coronary artery disease ${ }^{1-3)}$. SUA level is also known to have a prognostic value in patients with acute coronary syndrome (ACS), especially ST-segment elevation myocardial infarction $(\mathrm{MI})^{4,5}$. In addition, our recent investigation suggested that patients with ACS with elevated SUA level had greater lipid content of coronary plaque in non-culprit lesions, assessed by integrated backscatter intravascular ultrasound (IB-IVUS) ${ }^{6}$.

Previous reports have indicated that endothelial function plays an important role in the progression of coronary atherosclerosis ${ }^{7,8)}$. Significant relation between SUA level and endothelial function has been directly studied in patients with no overt cardiovascular dis- ease $^{9,10)}$, metabolic syndrome including hypertension and diabetes ${ }^{11)}$, and chronic kidney disease ${ }^{12)}$, primarily assessed by flow-mediated dilation (FMD). Although endothelial function is also considered to play an important role in the development of cardiovascular disease in patients with ACS with higher SUA level, their direct relation has not been well evaluated. Reactive hyperemia index (RHI) has been recently shown to be a useful method in non-invasively and reproducibly evaluating peripheral endothelial function ${ }^{13)}$, which has been validated to indicate the risk for cardiovascular adverse events ${ }^{14)}$. Additionally, RHI reportedly is operator-independent and easy to use compared with FMD ${ }^{15}$, indicating that RHI presents an alternative to FMD. The aim of this study was to investigate the relation between SUA level and endothelial function assessed by RHI in patients with ACS.

Address for correspondence: Yuichi Saito, Department of Cardiovascular Medicine, Chiba University Graduate School of Medicine, 1-8-1 Inohana, Chuo-ku, Chiba, Chiba 260-8677, Japan E-mail: policemaccho@yahoo.co.jp

Received: April 19, 2018 Accepted for publication: July 30, 2018 


\section{Methods}

\section{Study Population}

From December 2013 to November 2015, a total of 55 patients with ACS undergoing percutaneous coronary intervention (PCI) at Chiba University Hospital (Chiba, Japan) with measurements of both SUA level on admission and RHI were retrospectively enrolled in this study ${ }^{6}$. They were classified into three groups according to the tertiles of SUA level. The present study was conducted according to the Declaration of Helsinki. Written informed consent for examination was obtained from all patients, and the Ethical Committee of Chiba University approved the present study.

\section{Definition}

ACS was defined as unstable angina or acute MI developing $<48$ hours from the onset. The diagnosis of acute MI was according to the third universal definition of myocardial infarction ${ }^{16)}$. Unstable angina was diagnosed according to Braunwald's criteria with significant obstructive coronary artery disease ${ }^{17)}$. Although the PCI procedures were performed at the operator's discretion in the clinical settings, they were all performed under IVUS guidance. Hypertension, diabetes, and dyslipidemia were defined based on our previous report ${ }^{6)}$.

\section{Endothelial Function Assessment}

Endothelial function was evaluated by RHI using the EndoPAT 2000 device (Itamar Medical Inc., Caesarea, Israel), which has been validated to assess endothelial function with non-invasive, operator-independent, and reproducible procedure ${ }^{13,18,19)}$. The principle of measuring RHI has been previously described ${ }^{20,21)}$. Briefly, the patients fasted and refrained from caffeine, tobacco, and all types of medications for at least 8 hours. The measurement was performed in a quiet and temperature-controlled room early in the morning. A finger probe was placed on each index finger, and pulsatile volume changes in the distal digit were recorded by the EndoPAT 2000 device. After a 5-min baseline measurement, the cuff of blood pressure on the tested arm was inflated to obtain complete occlusion for $5 \mathrm{~min}$. Then, the cuff was deflated, and the EndoPAT 2000 device continued to trace peripheral arterial tonometry for another $5 \mathrm{~min}$. The data were automatically analyzed for calculation of RHI, which is the ratio of amplitude of the signal after cuff deflation divided by that before cuff inflation, indexed to the contralateral arm. RHI was measured on the day of discharge or a day earlier $(5.8 \pm 3.0$ days after PCI). On the same morning, trained nurses measured blood pressure of the patients lying in the supine position using an automated cuff sphygmomanometer (ESH55, Terumo, Tokyo, Japan).

\section{Statistical Analysis}

Statistical analysis was performed using SAS statistical software package version 9.4 (SAS Institute, Cary, NC). Data are presented as mean \pm SD or frequency (\%). Continuous variables were compared by analysis of variance and categorical variables with Fisher's exact test. The Kolmogorov-Smirnov test was used to examine the normal distribution of continuous variables. Univariate analysis for the variables in Table 1 was performed using linear regression analysis of rank-transformed outcomes. We included age, sex, and variables with a $p$-value of $<0.20$ on univariate analysis to conduct multivariate analysis by multiple linear regression analysis of rank-transformed outcomes. A $p$-value of $<0.05$ was considered statistically significant. Because this was a retrospective study, no power calculation was performed.

\section{Results}

Mean SUA level on admission and RHI were 5.8 $\pm 1.5 \mathrm{mg} / \mathrm{dl}$ and $1.88 \pm 0.58$, respectively. RHI was normally distributed $(p=0.36)$, and there was a significant negative correlation between SUA level and RHI $(r=-0.41, p=0.002)$. In addition, SUA level was negatively correlated with RHI $(r=-0.36, p=0.02)$ only in male patients $(n=42)$. The tertiles of SUA level were as follows: low tertile, $\leq 5.2 \mathrm{mg} / \mathrm{dl}$; intermediate tertile, 5.3 to $6.5 \mathrm{mg} / \mathrm{dl}$; and high tertile, $\geq 6.6 \mathrm{mg} / \mathrm{dl}$. Table 1 lists baseline characteristics of the patients. A higher proportion of males was observed in the high tertile group, and the patients in this group had greater body mass index and smaller estimate glomerular filtration rate compared with the other two groups. Additionally, the decrease of RHI was stepwisely observed in favor of the higher tertile groups $(2.14 \pm 0.74$ vs. $1.84 \pm 0.45$ vs. $1.67 \pm 0.38, p=0.04$; Fig.1). Multivariate analysis showed elevated SUA level as an independent predictor of RHI value (Table 2).

\section{Discussion}

The present study showed that SUA level was significantly associated with endothelial function assessed by RHI in patients with ACS. ACS with greater coronary plaque burden or lipid-rich components, advancing through endothelial dysfunction, has been recognized as an advanced phase of coronary atherosclerosis, and elevated SUA level is suggested to be 
Table 1. Patient Characteristics

\begin{tabular}{|c|c|c|c|c|}
\hline Variable & Low $(n=19)$ & Intermediate $(n=18)$ & High $(n=18)$ & $p$ value \\
\hline Age (years) & $69.2 \pm 8.7$ & $67.4 \pm 10.2$ & $64.1 \pm 15.3$ & 0.41 \\
\hline Men & $10(53 \%)$ & $15(83 \%)$ & $17(94 \%)$ & 0.007 \\
\hline Body mass index $\left(\mathrm{kg} / \mathrm{m}^{2}\right)$ & $22.6 \pm 3.5$ & $24.1 \pm 3.8$ & $25.6 \pm 3.7$ & 0.049 \\
\hline Hypertension & $15(79 \%)$ & $12(67 \%)$ & $16(89 \%)$ & 0.28 \\
\hline Diabetes mellitus & $6(32 \%)$ & $7(39 \%)$ & $4(22 \%)$ & 0.57 \\
\hline Dyslipidemia & $11(58 \%)$ & $15(83 \%)$ & $15(83 \%)$ & 0.12 \\
\hline Current smoker & $4(21 \%)$ & $8(44 \%)$ & $6(33 \%)$ & 0.33 \\
\hline Prior myocardial infarction & $2(11 \%)$ & $2(11 \%)$ & $2(11 \%)$ & $>0.99$ \\
\hline \multicolumn{5}{|l|}{ Clinical presentation } \\
\hline STEMI & $10(53 \%)$ & $10(56 \%)$ & $9(50 \%)$ & \multirow{3}{*}{0.72} \\
\hline NSTEMI & $5(26 \%)$ & $7(39 \%)$ & $7(39 \%)$ & \\
\hline Unstable angina & $4(21 \%)$ & $1(6 \%)$ & $2(11 \%)$ & \\
\hline eGFR $\left(\mathrm{ml} / \mathrm{min} / 1.73 \mathrm{~m}^{2}\right)$ & $78.9 \pm 18.1$ & $72.2 \pm 17.1$ & $58.6 \pm 18.5$ & 0.004 \\
\hline Serum uric acid (mg/dl) & $4.1 \pm 0.9$ & $5.8 \pm 0.4$ & $7.4 \pm 0.6$ & $<0.001$ \\
\hline \multicolumn{5}{|c|}{ Medical treatment on admission } \\
\hline ACE-I or ARB & $7(37 \%)$ & $6(33 \%)$ & $12(67 \%)$ & 0.09 \\
\hline$\beta$ blocker & $2(11 \%)$ & $2(11 \%)$ & $6(33 \%)$ & 0.13 \\
\hline Calcium channel blocker & $6(32 \%)$ & $5(28 \%)$ & $5(28 \%)$ & 0.96 \\
\hline Diuretic & $1(5 \%)$ & $2(11 \%)$ & $2(11 \%)$ & 0.78 \\
\hline Statin & $5(26 \%)$ & $4(22 \%)$ & $4(22 \%)$ & 0.95 \\
\hline Antihyperuricemic agent & $3(16 \%)$ & $1(6 \%)$ & $5(28 \%)$ & 0.20 \\
\hline
\end{tabular}

Patients were divided into 3 groups according to tertiles of SUA level. ACE-I=angiotensin converting enzyme inhibitor; $\mathrm{ARB}=$ angiotensin $I I$ receptor blocker; eGFR = estimate glomerular filtration rate; NSTEMI = non-ST elevation myocardial infarction; $\mathrm{STEMI}=\mathrm{ST}$ elevation myocardial infarction.

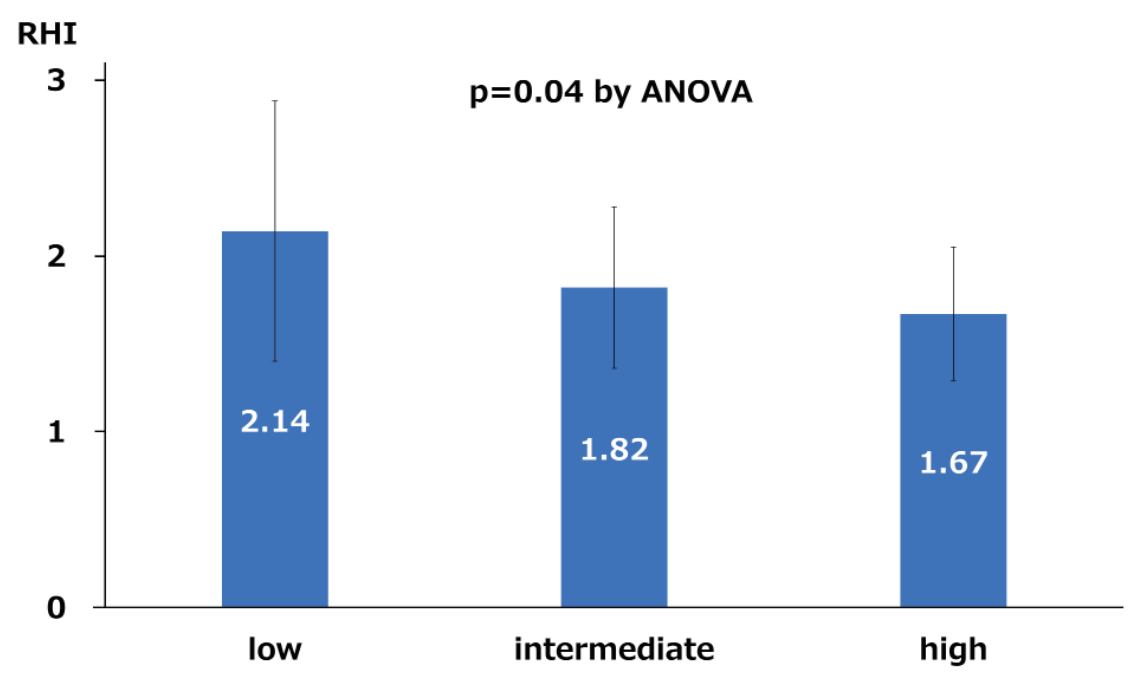

Fig. 1. Relation between RHI and serum uric acid levels divided by the tertiles of SUA level $\mathrm{RHI}$, reactive hyperemia index

related to the development of atherosclerosis and worse outcomes in patients with ACS. However, their direct relation has not been well studied. To the best of our knowledge, this is the first report investigating the direct relationship between SUA level and endothelial function in such subjects.
The endothelium regulates intravascular homeostasis, vasomotor tone, vascular permeability, inflammation, and smooth muscle cell proliferation and integrates numerous functions, such as blood pressure and coagulation ${ }^{22,23)}$. Endothelial dysfunction progresses the atherosclerotic changes within vascular wall 
Table 2. Predictors of reactive hyperemia index

\begin{tabular}{|c|c|c|c|c|}
\hline \multirow[t]{2}{*}{ Variable } & \multicolumn{2}{|c|}{ Univariate } & \multicolumn{2}{|c|}{ Multivariate } \\
\hline & $r$ & $p$ value & $\beta$ & $p$ value \\
\hline Age (years) & -0.11 & 0.44 & -0.25 & 0.14 \\
\hline Men & -0.16 & 0.24 & -0.02 & 0.88 \\
\hline Body mass index $\left(\mathrm{kg} / \mathrm{m}^{2}\right)$ & -0.06 & 0.66 & & \\
\hline Hypertension & 0.03 & 0.82 & & \\
\hline Diabetes mellitus & -0.12 & 0.38 & & \\
\hline Dyslipidemia & 0.16 & 0.25 & & \\
\hline Current smoker & -0.08 & 0.56 & & \\
\hline Prior myocardial infarction & 0.16 & 0.25 & & \\
\hline STEMI & 0.14 & 0.30 & & \\
\hline eGFR $\left(\mathrm{ml} / \mathrm{min} / 1.73 \mathrm{~m}^{2}\right)$ & 0.22 & 0.11 & -0.13 & 0.47 \\
\hline Serum uric acid (mg/dl) & -0.41 & 0.002 & -0.50 & 0.004 \\
\hline Systolic blood pressure (mm Hg) & -0.13 & 0.20 & & \\
\hline LDL cholesterol $(\mathrm{mg} / \mathrm{dl})$ & 0.11 & 0.50 & & \\
\hline ACE-I or ARB & 0.17 & 0.23 & & \\
\hline$\beta$ blocker & -0.25 & 0.07 & -0.14 & 0.29 \\
\hline Calcium channel blocker & -0.06 & 0.69 & & \\
\hline Diuretic & -0.01 & 0.97 & & \\
\hline Statin & 0.16 & 0.23 & & \\
\hline Antihyperuricemic agent & -0.09 & 0.50 & & \\
\hline
\end{tabular}

$\mathrm{eGFR}=$ estimated glomerular filtration rate; $\mathrm{LDL}=$ low-density lipoprotein.

with excessive production of reactive oxygen species and reduction of activity of endothelial nitric oxide synthase $^{23,24)}$. Although SUA has antioxidant capacities $^{25)}$, it is paradoxically known to have significant associations with inflammation and endothelial dysfunction ${ }^{9}$ 26). Xanthine oxidase $(\mathrm{XO})$, which catalyzes the metabolic reaction of converting purines to uric acid, simultaneously generates reactive oxygen species during the process ${ }^{23}, 27$ ), leading to endothelial dysfunction ${ }^{28}$. In clinical studies, the relation between SUA level and endothelial function has been also well investigated. Kato et al. demonstrated that endothelial function assessed by FMD inversely correlated with SUA level in 26 patients without any overt cardiovascular disease $(r=-0.4, p=0.05)^{9)}$. In a series of 263 patients with chronic kidney disease, Kanbay et al. similarly showed that the FMD value was negatively associated with SUA level $(r=-0.49, p<0.001)^{12)}$. The reported correlation coefficients between FMD values and SUA levels in various subjects were considerably varied ranging from -0.73 to $-0.26^{7,9,12,29-31)}$. Furthermore, endothelial function can be evaluated using $\mathrm{RHI}{ }^{19}{ }^{32)}$ in a non-invasive, reproducible, and operator-independent procedure ${ }^{13,18,19)}$. To date, there is only one report investigating the association of SUA level with RHI in subjects with metabolic syndrome, which also showed significant correlation between them $(r=-0.21, p<0.05)^{33)}$. In addition to endothe- lial function, the relation of SUA with vascular function was recently reported ${ }^{34)}$.

Previous studies have demonstrated that elevated SUA level is associated with cardiovascular events in patients with $\mathrm{ACS}^{4,5)}$. The retrospective analysis showed higher rates of in-hospital and long-term major adverse cardiovascular events in ST-segment elevation MI patients with high SUA level than in those with low SUA level ${ }^{5)}$. Cardiovascular mortality, reinfarction, target vessel revascularization, and severe heart failure were observed more frequently in the high SUA group than in the other groups. Increased cardiovascular mortality and reinfarction in patients with elevated SUA level might be partially explained by our previous report that demonstrated that these subjects had greater lipid content of coronary plaque assessed by IB-IVUS in non-culprit lesion ${ }^{6}$. However, the pathogenesis is partially understood. The present study indicated that the worse outcomes after ACS in patients with elevated SUA level are possibly associated with endothelial dysfunction because RHI has been reported to have prognostic values for cardiovascular events including cardiac death, MI, coronary revascularization, and heart failure ${ }^{14,35,36)}$. Recently, high XO activity with elevated SUA level in patients with chronic heart failure is reported to lead to cardiac events including cardiac death, ACS, and exacerbation heart failure requiring hospitalization ${ }^{37}$. Thus, ele- 
vated SUA level in patients with ACS might induce subsequent poor outcomes by endothelial dysfunction and XO activity. Randomized clinical trial of selective $\mathrm{XO}$ inhibitor to investigate whether it reduces adverse events is currently ongoing ${ }^{38)}$. Further study is needed to reconfirm the relation and elucidate whether reduction of SUA level or inhibition of XO recover endothelial function and contribute to the improvement of prognosis in patients with ACS.

There are some limitations to the present study. First, this is a single-center study and the number of included patients, especially women, was relatively small. Thus, the result in the present study was hypothesis-generating. Second, endothelial function was assessed by RHI in this study, whereas FMD has been previously used in many studies. Endothelial function assessment with non-invasive procedure has been traditionally and mainly performed by FMD over the recent 2 decades ${ }^{39)}$. However, operator dependence limited its use in clinical settings. Although the early report demonstrated a positive correlation between RHI and $\mathrm{FMD}^{40)}$, recent studies did not ${ }^{32)}$. Nevertheless, RHI, representing digital microvessel dilation, is considered to assess endothelial function as FMD, which assesses conduit artery vasodilation ${ }^{32)}$. RHI and FMD measure distinct components of vascular health, whereas RHI and FMD are reported to predict cardiovascular events with similar prognostic magnitude ${ }^{19)}$. Third, patients with ACS may be affected by many factors such as hemodynamic change or recently initiated medication following ACS. However, RHI was measured under the stabilized condition on the day of discharge or a day earlier early in the morning without any medications for at least 8 hours ${ }^{21)}$. Lastly, although inflammation reaction and oxidative stress are the key factors in the process of endothelial function, serum inflammation factor (e.g., high-sensitivity C-reactive protein, interleukin-6, and tumor necrosis factor- $\alpha$ ) and oxidative stress marker (e.g., isoprostanes, 8-hydroxy-2' -deoxyguanosine, and thiobarbituric acid reactive substances) were not evaluated in the present study. In conclusion, elevated SUA level was significantly associated with endothelial dysfunction in patients with ACS, possibly leading to subsequent poor outcomes following ACS.

\section{Disclosure}

None.

\section{References}

1) Bos MJ, Koudstaal PJ, Hofman A, Witteman JC, Breteler MM: Uric acid is a risk factor for myocardial infarction and stroke: the Rotterdam study. Stroke, 2006; 37: 15031507

2) Kaya EB, Yorgun H, Canpolat U, Hazırolan T, Sunman H, Ülgen A, Ates AH, Aytemir K, Tokgözoğlu L, Kabakc1 G, Akata D, Oto A: Serum uric acid levels predict the severity and morphology of coronary atherosclerosis detected by multidetector computed tomography. Atherosclerosis, 2010; 213: 178-183

3) Sinan Deveci O, Kabakci G, Okutucu S, Tulumen E, Aksoy H, Baris Kaya E, Evranos B, Aytemir K, Tokgozoglu L, Oto A: The association between serum uric acid level and coronary artery disease. Int J Clin Pract, 2010; 64: 900-907

4) Kojima S, Sakamoto T, Ishihara M, Kimura K, Miyazaki S, Yamagishi M, Tei C, Hiraoka H, Sonoda M, Tsuchihashi K, Shimoyama N, Honda T, Ogata Y, Matsui K, Ogawa H; Japanese Acute Coronary Syndrome Study (JACSS) Investigators: Prognostic usefulness of serum uric acid after acute myocardial infarction (the Japanese Acute Coronary Syndrome Study). Am J Cardiol, 2005; 96: 489-495

5) Kaya MG, Uyarel H, Akpek M, Kalay N, Ergelen M, Ayhan E, Isik T, Cicek G, Elcik D, Sahin O, Cosgun SM, Oguzhan A, Eren M, Gibson CM: Prognostic value of uric acid in patients with ST-elevated myocardial infarction undergoing primary coronary intervention. Am J Cardiol, 2012; 109: 486-491

6) Saito Y, Nakayama T, Sugimoto K, Fujimoto Y, Kobayashi Y: Relation of Lipid Content of Coronary Plaque to Level of Serum Uric Acid. Am J Cardiol, 2015; 116: 13461350

7) Mercuro G, Vitale C, Cerquetani E, Zoncu S, Deidda M, Fini M, Rosano GM: Effect of hyperuricemia upon endothelial function in patients at increased cardiovascular risk. Am J Cardiol, 2004; 94: 932-935

8) Feig DI, Kang DH, Johnson RJ: Uric acid and cardiovascular risk. N Engl J Med, 2008; 359: 1811-1821

9) Kato M, Hisatome I, Tomikura Y, Kotani K, Kinugawa T, Ogino K, Ishida K, Igawa O, Shigemasa C, Somers VK: Status of endothelial dependent vasodilation in patients with hyperuricemia. Am J Cardiol, 2005; 96: 1576-1578

10) Ho WJ, Tsai WP, Yu KH, Tsay PK, Wang CL, Hsu TS, Kuo CT: Association between endothelial dysfunction and hyperuricaemia. Rheumatology (Oxford), 2010; 49: 1929-1934

11) Tomiyama H, Higashi $Y$, Takase B, Node $K$, Sata M, Inoue T, Ishibashi Y, Ueda S, Shimada K, Yamashina A: Relationships among hyperuricemia, metabolic syndrome, and endothelial function. Am J Hypertens, 2011; 24: 770-774

12) Kanbay M, Yilmaz MI, Sonmez A, Turgut F, Saglam M, Cakir E, Yenicesu M, Covic A, Jalal D, Johnson RJ: Serum uric acid level and endothelial dysfunction in patients with nondiabetic chronic kidney disease. Am J Nephrol, 2011; 33: 298-304

13) Selamet Tierney ES, Newburger JW, Gauvreau K, Geva J, Coogan E, Colan SD, de Ferranti SD: Endothelial pulse amplitude testing: feasibility and reproducibility in adolescents. J Pediatr, 2009; 154: 901-905

14) Rubinshtein R, Kuvin JT, Soffler M, Lennon RJ, Lavi S, Nelson RE, Pumper GM, Lerman LO, Lerman A: Assess- 
ment of endothelial function by non-invasive peripheral arterial tonometry predicts late cardiovascular adverse events. Eur Heart J, 2010; 31: 1142-1148

15) Flammer AJ, Anderson T, Celermajer DS, Creager MA, Deanfield J, Ganz P, Hamburg NM, Lüscher TF, Shechter M, Taddei S, Vita JA, Lerman A: The assessment of endothelial function: from research into clinical practice. Circulation, 2012; 126: 753-767

16) Thygesen K, Alpert JS, Jaffe AS, Simoons ML, Chaitman BR, White HD: Third universal definition of myocardial infarction. J Am Coll Cardiol, 2012; 60: 1581-1598

17) Braunwald E: Unstable angina. A classification. Circulation, 1989; 80: 410-414

18) Bonetti PO, Pumper GM, Higano ST, Holmes DR Jr, Kuvin JT, Lerman A: Noninvasive identification of patients with early coronary atherosclerosis by assessment of digital reactive hyperemia. J Am Coll Cardiol, 2004; 44: 2137-2141

19) Matsuzawa Y, Kwon TG, Lennon RJ, Lerman LO, Lerman A: Prognostic Value of Flow-Mediated Vasodilation in Brachial Artery and Fingertip Artery for Cardiovascular Events: A Systematic Review and Meta-Analysis. J Am Heart Assoc, 2015; 4: e002270

20) Axtell AL, Gomari FA, Cooke JP: Assessing endothelial vasodilator function with the Endo-PAT 2000. J Vis Exp, 2010; doi: $10.3791 / 2167$

21) Saito $Y$, Kitahara H, Nakayama T, Fujimoto Y, Kobayashi Y: Night-time blood pressure variability negatively correlated with reactive hyperemia index. Int J Cardiol, 2017; 230: 332-334

22) Higashi Y, Noma K, Yoshizumi M, Kihara Y: Endothelial function and oxidative stress in cardiovascular diseases. Circ J, 2009; 73: 411-418

23) Okafor ON, Farrington K, Gorog DA: Allopurinol as a therapeutic option in cardiovascular disease. Pharmacol Ther, 2017; 172: 139-150

24) Förstermann U, Münzel T: Endothelial nitric oxide synthase in vascular disease: from marvel to menace. Circulation, 2006; 113: 1708-1714

25) Glantzounis GK, Tsimoyiannis EC, Kappas AM, Galaris DA: Uric acid and oxidative stress. Curr Pharm Des, 2005; 11: 4145-4151

26) Ruggiero C, Cherubini A, Ble A, Bos AJ, Maggio M, Dixit VD, Lauretani F, Bandinelli S, Senin U, Ferrucci L: Uric acid and inflammatory markers. Eur Heart J, 2006; 27: 1174-1181

27) George J, Struthers AD: Role of urate, xanthine oxidase and the effects of allopurinol in vascular oxidative stress. Vasc Health Risk Manag, 2009; 5: 265-272

28) Nieto FJ, Iribarren C, Gross MD, Comstock GW, Cutler RG: Uric acid and serum antioxidant capacity: a reaction to atherosclerosis? Atherosclerosis, 2000; 148: 131-139

29) Kanbay M, Huddam B, Azak A, Solak Y, Kadioglu GK, Kirbas I, Duranay M, Covic A, Johnson RJ: A randomized study of allopurinol on endothelial function and estimated glomular filtration rate in asymptomatic hyperuricemic subjects with normal renal function. Clin J Am Soc Nephrol, 2011; 6: 1887-1894

30) Erdogan D, Gullu H, Caliskan M, Yildirim E, Bilgi M, Ulus T, Sezgin N, Muderrisoglu H: Relationship of serum uric acid to measures of endothelial function and athero- sclerosis in healthy adults. Int J Clin Pract, 2005; 59: 1276-1282

31) Maxwell AJ, Bruinsma KA: Uric acid is closely linked to vascular nitric oxide activity. Evidence for mechanism of association with cardiovascular disease. J Am Coll Cardiol, 2001; 38: 1850-1858

32) Hamburg NM, Palmisano J, Larson MG, Sullivan LM, Lehman BT, Vasan RS, Levy D, Mitchell GF, Vita JA, Benjamin EJ: Relation of brachial and digital measures of vascular function in the community: the Framingham heart study. Hypertension, 2011; 57: 390-396

33) Kwaśniewska M, Kozińska J, Dziankowska-Zaborszczyk E, Kostka T, Jegier A, Rębowska E, Orczykowska M, Leszczyńska J, Drygas W: The impact of long-term changes in metabolic status on cardiovascular biomarkers and microvascular endothelial function in middle-aged men: a 25-year prospective study. Diabetol Metab Syndr, 2015; 7: 81

34) Liu H, Liu J, Zhao H, Zhou Y, Li L, Wang H; BEST Research Group: Relationship between Serum Uric Acid and Vascular Function and Structure Markers and Gender Difference in a Real-World Population of China-From Beijing Vascular Disease Patients Evaluation Study (BEST) Study. J Atheroscler Thromb, 2018; 25: 254-261

35) Reriani MK, Flammer AJ, Jama A, Lerman LO, Lerman A: Novel functional risk factors for the prediction of cardiovascular events in vulnerable patients following acute coronary syndrome. Circ J, 2012; 76: 778-783

36) Matsuzawa Y, Sugiyama S, Sumida H, Sugamura K, Nozaki T, Ohba K, Matsubara J, Kurokawa H, Fujisue K, Konishi M, Akiyama E, Suzuki H, Nagayoshi Y, Yamamuro M, Sakamoto K, Iwashita S, Jinnouchi H, Taguri M, Morita S, Matsui K, Kimura K, Umemura S, Ogawa $\mathrm{H}$ : Peripheral endothelial function and cardiovascular events in high-risk patients. J Am Heart Assoc, 2013; 2: e000426

37) Otaki Y, Watanabe T, Kinoshita D, Yokoyama M, Takahashi T, Toshima T, Sugai T, Murase T, Nakamura T, Nishiyama S, Takahashi H, Arimoto T, Shishido T, Miyamoto T, Kubota I: Association of plasma xanthine oxidoreductase activity with severity and clinical outcome in patients with chronic heart failure. Int J Cardiol, 2017; 228: $151-157$

38) Kojima S, Matsui K, Ogawa H, Jinnouchi H, Hiramitsu S, Hayashi T, Yokota N, Kawai N, Tokutake E, Uchiyama K, Sugawara M, Kakuda H, Wakasa Y, Mori H, Hisatome I, Waki M, Ohya Y, Kimura K, Saito Y; Febuxostat for Cerebral and Cardiorenovascular Events Prevention Study (FREED) investigators: Rationale, design, and baseline characteristics of a study to evaluate the effect of febuxostat in preventing cerebral, cardiovascular, and renal events in patients with hyperuricemia. J Cardiol, 2017; 69: 169-175

39) Sauder KA, West SG, McCrea CE, Campbell JM, Jenkins AL, Jenkins DJ, Kendall CW: Test-retest reliability of peripheral arterial tonometry in the metabolic syndrome. Diab Vasc Dis Res, 2014; 11: 201-207

40) Kuvin JT, Patel AR, Sliney KA, Pandian NG, Sheffy J, Schnall RP, Karas RH, Udelson JE: Assessment of peripheral vascular endothelial function with finger arterial pulse wave amplitude. Am Heart J, 2003; 146: 168-174 